\title{
On Critical Circle Homeomorphisms with Infinite Number of Break Points
}

\author{
Akhtam Dzhalilov, ${ }^{1}$ Mohd Salmi Md Noorani, ${ }^{2}$ and Sokhobiddin Akhatkulov ${ }^{2}$ \\ ${ }^{1}$ Turin Polytechnic University, Kichik Halka Yuli 17, 100095 Tashkent, Uzbekistan \\ ${ }^{2}$ School of Mathematical Sciences, Faculty of Science and Technology, University Kebangsaan Malaysia (UKM), 43600 Bangi, \\ Selangor Darul Ehsan, Malaysia
}

Correspondence should be addressed to Sokhobiddin Akhatkulov; akhatkulov@yahoo.com

Received 4 October 2013; Accepted 30 January 2014; Published 20 March 2014

Academic Editor: Weinian Zhang

Copyright (C) 2014 Akhtam Dzhalilov et al. This is an open access article distributed under the Creative Commons Attribution License, which permits unrestricted use, distribution, and reproduction in any medium, provided the original work is properly cited.

We prove that a critical circle homeomorphism with infinite number of break points without periodic orbits is conjugated to the linear rotation by a quasisymmetric map if and only if its rotation number is of bounded type. And we also prove that any two adjacent atoms of dynamical partition of a unit circle are comparable.

\section{Introduction}

Let $S^{1}=\mathbb{R} / \mathbb{Z}$ with clearly defined orientation, metric, Lebesgue measure, and the operation of addition be the unit circle. Let $\pi: \mathbb{R} \rightarrow S^{1}$ denote the corresponding projection mapping that "winds" a straight line on the circle. An arbitrary homeomorphism $f$ that preserves the orientation of the unit circle $S^{1}$ can "be lifted" on the straight line $\mathbb{R}$ in the form of the homeomorphism $F: \mathbb{R} \rightarrow \mathbb{R}$ with property $F(x+1)=F(x)+1$ that is connected with $f$ by relation $\pi \circ F=f \circ \pi$. This homeomorphism $F$ is called the lift of the homeomorphism $f$ and is defined up to an integer term. The most important arithmetic characteristic of the homeomorphism $f$ of the unit circle $S^{1}$ is the rotation number:

$$
\rho(f)=\lim _{n \rightarrow \infty} \frac{F^{n}(x)}{n} \bmod 1,
$$

where $F$ is the lift of $f$ with $S^{1}$ to $\mathbb{R}$. Here and below, for a given map $F, F^{n}$ denotes its $n$th iterate. The rotation number is rational if and only if $f$ has periodic points. Denjoy proved that if $f$ is a circle diffeomorphism with irrational rotation number $\rho=\rho(f)$ and $\log D f$ is of bounded variation, then $f$ is topologically conjugate to the pure rotation $f_{\rho}$ : $x \rightarrow x+\rho \bmod 1$; that is, there exists an essentially unique homeomorphism $\varphi$ of the circle with $\varphi \circ f=f_{\rho} \circ \varphi$ (see [1]). Since the conjugating map $\varphi$ and the unique $f$ invariant measure $\mu_{f}$ are related by $\varphi(x)=\mu_{f}([0, x]$ ) (see [1]), regularity properties of the conjugating map $\varphi$ imply corresponding properties of the density of the absolutely continuous invariant measure $\mu_{f}$. The problem of relating the smoothness of $\varphi$ to that of $f$ has been studied extensively. Indepth results have been found; see [2-5].

Other classes of circle homeomorphisms are known to satisfy the conclusion of Denjoy's theorem (see [6], Chapters I and IV, and [2], Chapter VI) and the study of the regularity of their conjugation maps arises naturally. Two of these classes are commonly referred to as the following.

1.1. Critical Circle Homeomorphisms. The orientation preserving circle homeomorphisms $f$, such that $f \in C^{r}, r \geq$ 3 , have finite number of critical points $c_{i}$, around which, in some $C^{r}$ coordinate system, $f$ has the form $x \rightarrow x^{p_{i}}$, where $p_{i} \geq 3$ are the odd integers. Such critical points we say are of polynomial type of order $p_{i}$.

1.2. P-Homeomorphisms. That is, orientation preserving circle homeomorphisms $f$ are differentiable except in many countable points called break points admitting left and right derivatives (denoted by $D f_{-}$and $D f_{+}$, resp.) such that 
(i) there exist some constants $0<\gamma<\zeta<\infty$ such that $\gamma<D f(x)<\zeta$ for all $x \in S^{1} \backslash B P(f), \gamma<D f_{+}(b)<\zeta$ and $\gamma<D f_{-}(b)<\zeta$ for all $b \in B P(f)$, where $B P(f)$ denotes the set of the break points of $f$;

(ii) $\log D f$ has bounded variation. In this situation, $\log D f, \log D f_{-}, \log D f_{+}$and $\log D f^{-1}, \log D f_{-}^{-1}$, $\log D f_{+}^{-1}$ have the same total variation denoted by $V=\operatorname{Var} \log D f$.

The ratio $\sigma_{f}(b)=D f_{-}(b) / D f_{+}(b)$ is called jump ratio of $f$ in $b$.

The existence of the conjugating map for the class critical circle homeomorphisms was proved by Yoccoz in [7] and for the class $P$-homeomorphisms the existence of conjugating map was proved by Herman in [2].

The singularity of the conjugating map for critical circle homeomorphisms was shown by Graczyk and Świątek in [8]. They proved that if $f$ is $C^{3}$ smooth circle homeomorphism with finitely many critical points of polynomial type and an irrational rotation number of bounded type, then the conjugating map $\varphi$ is a singular function. For the $P$ homeomorphisms, the situation is different; that is, in this case, the conjugating map can be singular or absolutely continuous. Indeed, in the works [9-11], it was shown that the conjugating map is singular. The deeper result in this area was obtained by Dzhalilov et al. [12]. They proved that if $f$ is piecewise-smooth $P$-homeomorphism with finite number of break points and the product of jump ratios at these break points is nontrivial, then the conjugating map is a singular function. But in the works $[9,13]$, it was shown that if $f$ is piecewise-smooth $P$-homeomorphism with finite number of break points having the (D)-property (see for the definition [13]) and the product of the jump ratios on each orbit is equal to 1 , then the conjugating map is an absolutely continuous function. Now, we discuss the symmetric property of a given function.

Definition 1. A homeomorphism $g: \mathbb{R} \rightarrow \mathbb{R}$ is called Qquasisymmetric if and only if, for every real $x$ and $\delta \neq 0$,

$$
\frac{|g(x+\delta)-g(x)|}{|g(x)-g(x-\delta)|} \leq Q .
$$

The criteria of quasisymmetry of the conjugating map of the critical circle homeomorphisms were obtained by Świątek in [14]. Due to [14], if the circle homeomorphism with an irrational rotation number is analytic and has finitely many critical points, then the conjugating map is quasisymmetric if and only if the rotation number is of bounded type.

The quasisymmetric property of the conjugating map of $P$-homeomorphisms is also different from the case of critical circle homeomorphisms. More precisely, if the rotation number of $P$-homeomorphism is irrational of bounded type, then conjugating map is quasisymmetric, but there is a $P$-homeomorphism with irrational rotation number of unbounded type such that the conjugating map is quasisymmetric. In this paper, we introduce a new class of circle homeomorphisms with the aid of the above two classes. Our aim in this work is to show the existence of conjugating map for this new class and study the quasisymmetric property of this conjugating map. Now, we introduce our class.

Let $f$ be a circle homeomorphism.

(a) $f$ has one critical point polynomial type of order 3; that is, there is a point $x=x_{c r} \in S^{1}$ such that $D f\left(x_{c r}\right)=D^{2} f\left(x_{c r}\right)=0$ and $D^{3} f\left(x_{c r}\right) \neq 0$.

(b) $f \in C^{3}\left(U_{\epsilon}\left(x_{c r}\right)\right)$ for some $\epsilon$-neighborhood of the point $x_{c r}$.

(c) $f$ is a $P$-homeomorphism on the set $S^{1} \backslash U_{\epsilon}\left(x_{c r}\right)$.

Note that all the above results were obtained for the class $P$-homeomorphisms with finite number of break points, but in our work it is not necessary for the number of break points to be finite. Now, we state our main results.

Theorem 2. Suppose that a circle homeomorphism $f$ satisfies the conditions $(a)-(c)$ and the rotation number $\rho(f)$ is irrational. Then, there exists circle homeomorphism $H: S^{1} \rightarrow S^{1}$, such that the functional equation

$$
H(x+\rho(f))=f(H(x))
$$

is satisfied. Moreover, $H$ is quasisymmetric if and only if $\rho(f)$ is of bounded type.

The proof of Theorem 2 is based on the method of crossratio distortion estimates. Note that the cross-ratio estimates were used in dynamical systems for the first time by Yoccoz [7] and later by Świątek [14]. In fact, the proof of Theorem 2 follows closely that of Świątek [14]. Our second result below is also proved by using cross-ratio estimates.

Theorem 3. Suppose that a circle homeomorphism $f$ satisfies the conditions $(a)-(c)$ and the rotation number $\rho(f)$ is irrational. Then, there exists universal constant $C=C(f)>1$ such that any two adjacent atoms $I_{1}$ and $I_{2}$ of a dynamical partition $\mathbf{P}_{n}\left(x_{c r}, f\right)$ (see, for the definition, below) are C-comparable; that is,

$$
C^{-1}\left|I_{2}\right| \leq\left|I_{1}\right| \leq C\left|I_{2}\right|
$$

\section{Dynamical Partition}

We will assume that the rotation number $\rho=\rho(f)$ is irrational throughout this paper. We use the continued fraction representation $\rho=1 /\left(k_{1}+1 /\left(k_{2}+\cdots\right)\right):=\left[k_{1}, k_{2}, \ldots, k_{n}, \ldots\right)$ of the rotation number, which is understood as a limit of the sequence of convergents $p_{n} / q_{n}=\left[k_{1}, k_{2}, \ldots, k_{n}\right]$. The sequence of positive integers $k_{n}$ with $n \geq 1$, which are called partial quotients, are uniquely determined for each $\rho$. The coprimes $p_{n}$ and $q_{n}$ satisfy the recurrence relations $p_{n}=$ $k_{n} p_{n-1}+p_{n-2}$ and $q_{n}=k_{n} q_{n-1}+q_{n-2}$ for $n \geq 1$, where we set, for convenience, $p_{-1}=0, q_{-1}=1$ and $p_{0}=1, q_{0}=k_{1}$. For $\xi \in S^{1}$ we define the $n$th fundamental segment $I_{0}^{n}=I_{0}^{n}(\xi)$ as the circle arc $\left[\xi, f^{q_{n}}(\xi)\right]$ if $n$ is even and $\left[f^{q_{n}}(\xi), \xi\right]$ if $n$ is odd. We denote two sets of closed intervals of order $n: q_{n}$ "long" intervals: $\left\{I_{i}^{n-1}=f^{i}\left(I_{0}^{n-1}\right), 0 \leq i<q_{n}\right\}$ and $q_{n-1}$ "short" intervals: $\left\{I_{j}^{n}=f^{j}\left(I_{0}^{n}\right), 0 \leq j<q_{n-1}\right\}$. The long and short 
intervals are mutually disjoint except for the endpoints and cover the whole circle. The partition obtained by the above construction will be denoted by $\mathbf{P}_{n}=\mathbf{P}_{n}(\xi, f)$ and called the $n$th dynamical partition of $S^{1}$. Obviously, the partition $\mathbf{P}_{n+1}$ is a refinement of the partition $\mathbf{P}_{n}$. Indeed, the short intervals are members of $\mathbf{P}_{n+1}$ and each long interval $I_{i}^{n-1} \in \mathbf{P}_{n}, 0 \leq$ $i<q_{n}$, is partitioned into $k_{n+1}+1$ intervals belonging to $\mathbf{P}_{n+1}$ such that

$$
I_{i}^{n-1}=I_{i}^{n+1} \cup \bigcup_{s=0}^{k_{n+1}-1} I_{i+q_{n-1}+s q_{n}}^{n} .
$$

\section{Cross-Ratio Inequality}

Now, we equip $S^{1}$ with the usual metric $|x-y|=\inf \{|\tilde{x}-\tilde{y}|$, where $\tilde{x}, \tilde{y}$ range over the lifts of $x, y \in S^{1}$, resp. $\}$. Our main analytic tools are ratio and cross-ratio distortions. Let $a, b, c, d \in S^{1}$ be four points of the circle which preserve orientation; that is, $a \prec b \prec c \prec d \prec a$ on the circle; we define a ratio of three points of $a, b, c$ by

$$
R(a, b, c)=\frac{|b-a|}{|c-a|},
$$

and we define a cross-ratio of four points of $a, b, c, d$ by

$$
\operatorname{Cr}(a, b, c, d)=\frac{|b-a|}{|c-a|} \cdot \frac{|d-c|}{|d-b|} .
$$

The distortions $D r$ and $D c r$ of the ratio and the cross-ratio by the function $f$ are defined by

$$
\begin{gathered}
\operatorname{Dr}(a, b, c ; f)=\frac{R(f(a), f(b), f(c))}{R(a, b, c)}, \\
\operatorname{Dcr}(a, b, c, d ; f)=\frac{\operatorname{Cr}(f(a), f(b), f(c), f(d))}{\operatorname{Cr}(a, b, c, d)},
\end{gathered}
$$

respectively. It is clear that

$$
\operatorname{Dcr}(a, b, c, d ; f)=\operatorname{Dr}(a, b, c ; f) \cdot \operatorname{Dr}(d, c, b ; f) .
$$

Notice that the ratio and the cross-ratio distortions have the following properties.

(i) The ratio and the cross-ratio are invariant by translation:

$$
\begin{gathered}
R(a+t, b+t, c+t)=R(a, b, c), \\
C r(a+t, b+t, c+t, d+t)=\operatorname{Cr}(a, b, c, d), \quad t \in S^{1} .
\end{gathered}
$$

(ii) The ratio and the cross-ratio distortions are multiplicative with respect to composition for two functions $F$ and $G$ on $S^{1}$; we have

$$
\begin{aligned}
\operatorname{Dr}(a, b, c ; F \circ G)= & \operatorname{Dr}(G(a), G(b), G(c) ; F) \\
& \cdot \operatorname{Dr}(a, b, c ; G), \\
\operatorname{Dcr}(a, b, c, d ; F \circ G)= & \operatorname{Dcr}(G(a), G(b), G(c), G(d) ; F) \\
& \cdot \operatorname{Dcr}(a, b, c, d ; G) .
\end{aligned}
$$

Now, let us formulate the following theorem, which plays an important role during studying the properties of dynamical partitions.

Theorem 4. Suppose that a homeomorphism $f$ satisfies the conditions $(a)-(c)$. Consider a system of quadruples $\left\{\left(a_{i}, b_{i}, c_{i}, d_{i}\right), a_{i} \prec b_{i} \prec c_{i} \prec d_{i} \prec a_{i}, 1 \leq i \leq m\right\}$ on the circle $S^{1}$. Suppose that the system of intervals $\left\{\left(a_{i}, d_{i}\right), 1 \leq i \leq m\right\}$ covers each point of the circle at most $p$ times. Then, there exists a constant $Q_{1}=Q_{1}(p, f)$ such that the following inequality holds:

$$
\prod_{i=1}^{m} \operatorname{Dcr}\left(a_{i}, b_{i}, c_{i}, d_{i} ; f\right) \leq Q_{1} .
$$

Inequality (12) is called the cross-ratio inequality. This inequality was proved for the critical circle homeomorphisms by Świątek [15]. Now, we prove the following three important lemmas to be used in the proof of the main results.

Lemma 5. Suppose that a homeomorphism $f$ satisfies the conditions (a)-(c). Consider a system of quadruples $\left\{\left(a_{i}, b_{i}, c_{i}, d_{i}\right)\right.$, $\left.a_{i} \prec b_{i} \prec c_{i} \prec d_{i} \prec a_{i}, 1 \leq i \leq m\right\}$ on the circle $S^{1}$. Suppose that the system of intervals $\left\{\left(a_{i}, d_{i}\right), 1 \leq i \leq m\right\}$ covers each point of the set $S^{1} \backslash U_{\epsilon}\left(x_{c r}\right)$ at most $p$ times. Then, there exists a constant $Q_{2}=Q_{2}(f, p)$ such that the following inequality holds:

$$
\prod_{i=1}^{m} \operatorname{Dcr}\left(a_{i}, b_{i}, c_{i}, d_{i} ; f\right) \leq Q_{2} .
$$

Proof. Since $f$ is a $P$-homeomorphism on the set $S^{1} \backslash U_{\epsilon}\left(x_{c r}\right)$ for any quadruples $\left(a_{i}, b_{i}, c_{i}, d_{i}\right)$ satisfying assumption of lemma, we have

$$
\begin{aligned}
\left|\log D r\left(a_{i}, b_{i}, c_{i} ; f\right)\right| & \leq \sup _{x, y \in\left[a_{i}, c_{i}\right]}|\log D f(x)-\log D f(y)| \\
& \leq \underset{\left[a_{i}, c_{i}\right]}{\operatorname{Var} \log D f .}
\end{aligned}
$$

Using this inequality, we get

$$
\begin{aligned}
\left|\log \prod_{i=1}^{m} \operatorname{Dcr}\left(a_{i}, b_{i}, c_{i}, d_{i} ; f\right)\right| & \leq \sum_{i=1}^{m}\left|\log \operatorname{Dcr}\left(a_{i}, b_{i}, c_{i}, d_{i} ; f\right)\right| \\
& \leq 2 \sum_{i=1}^{m}\left[a_{i}, d_{i}\right]
\end{aligned}
$$

By assumption, the system of intervals $\mathfrak{F}:=\left\{\left[a_{i}, d_{i}\right], 1 \leq\right.$ $i \leq m\}$ covers each point of the set $S^{1} \backslash U_{\epsilon}\left(x_{c r}\right)$ at most $p$ times. Now, we describe this system of intervals as a union of subsystems of $\mathfrak{F}_{j}, j \leq p$ in the following way: first, we take $\left[a_{1}, d_{1}\right]$ as an element of $\mathfrak{F}_{1}$ and then consider the intersection $\left[a_{1}, d_{1}\right] \bigcap\left[a_{2}, d_{2}\right]$; if this intersection is empty, then we count the interval $\left[a_{2}, d_{2}\right]$ an element of $\mathfrak{F}_{1}$; otherwise, we count an element of $\mathfrak{F}_{2}$. Next, consider $\mathfrak{F}_{1} \bigcap\left[a_{3}, d_{3}\right]$ (here and below, it is considered the intersection with each element 
of $\left.\mathfrak{F}_{1}\right)$; if it is empty, we count $\left[a_{3}, d_{3}\right]$ an element of $\mathfrak{F}_{1}$; otherwise, we check the intersection $\mathfrak{F}_{2} \bigcap\left[a_{3}, d_{3}\right]$. Again, if $\mathfrak{F}_{2} \bigcap\left[a_{3}, d_{3}\right]$ is empty, we count $\left[a_{3}, d_{3}\right]$ an element of $\mathfrak{F}_{2}$; otherwise, we count $\left[a_{3}, d_{3}\right]$ an element of $\mathfrak{F}_{3}$. Continuing this process, we get all $\mathfrak{F}_{j}, j \leq p$. By construction of subsystems $\mathfrak{F}_{j}, j \leq p$ of $\mathfrak{F}$, the elements of each subsystem do not intersect with each other and $\mathfrak{F}=\bigcup_{j} \mathfrak{F}_{j}, j \leq p$. Therefore

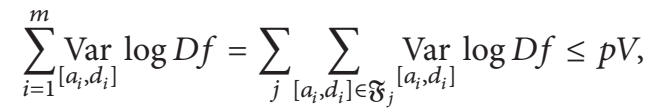

where $V=\underset{S^{1} \backslash U_{\epsilon}\left(x_{c r}\right)}{\operatorname{Var}} \log D f$.

We will use the following definition and fact to formulate the next lemma.

Definition 6. Let $g$ be a $C^{3}$ function such that $D g \neq 0$. The Schwarzian derivative of $g$ is defined by

$$
\delta g=\frac{D^{3} g}{D g}-\frac{3}{2}\left(\frac{D^{2} g}{D g}\right)^{2}
$$

Fact (see [6]). If $\mathcal{S} f<0$ on interval $I$, then

$$
\operatorname{Dcr}(a, b, c, d ; f)<1
$$

for any quadruples $a, b, c, d \in I$.

Lemma 7. Suppose that a circle homeomorphism $f$ satisfies the conditions $(a)-(c)$. Then, there is $\epsilon_{0} \in(0, \epsilon / 2)$, such that $f(x)$ satisfies the following conditions:

(i) $\mathcal{S} f<0$ in $U_{2 \epsilon_{0}}\left(x_{c r}\right) \backslash\left\{x_{c r}\right\}$;

(ii) there exist constants $0<Q_{3} \leq Q_{4}$, such that $Q_{3}(x-$ $\left.x_{c r}\right)^{2} \leq D f(x) \leq Q_{4}\left(x-x_{c r}\right)^{2}$ for all $x \in U_{2 \epsilon_{0}}\left(x_{c r}\right)$.

Proof. Consider the following function:

$$
v(x)=\frac{D^{3} f(x)}{2}, \quad x \in U_{\epsilon}\left(x_{c r}\right) .
$$

By using Taylor's formula, we have

$$
D f(x)=v\left(x_{1}\right)\left(x-x_{c r}\right)^{2}, \quad D^{2} f(x)=2 v\left(x_{2}\right)\left(x-x_{c r}\right),
$$

where $\left|x_{i}-x_{c r}\right| \leq\left|x-x_{c r}\right|, i=1,2$. Since $v(x)$ is a nonzero, continuous function and if we take $Q_{3}=\inf _{x \in U_{\epsilon}\left(x_{c r}\right)} v(x)$, $Q_{4}=\sup _{x \in U_{\epsilon}\left(x_{c r}\right)} v(x)$, then the second assertion of this lemma follows. In order to prove the first assertion of this lemma we calculate the Schwarzian derivative of the function $f(x)$ as follows:

$$
\mathcal{S} f(x)=\frac{2}{\left(x-x_{c r}\right)^{2}}\left[\frac{v(x)}{v\left(x_{1}\right)}-3\left(\frac{v\left(x_{2}\right)}{v\left(x_{1}\right)}\right)^{2}\right] .
$$

Since $v(x)$ is nonzero and continuous on $U_{\epsilon}\left(x_{c r}\right)$, there is a sufficient small $\epsilon_{0} \in(0, \epsilon / 2)$ such that the ratios $v(x) / v\left(x_{1}\right)$ and $v\left(x_{2}\right) / v\left(x_{1}\right)$ are uniformly close to 1 for any $x, x_{1}, x_{2} \in$ $U_{2 \epsilon_{0}}\left(x_{c r}\right)$. It ensures that $\mathcal{S} f(x)<0, x \in U_{2 \epsilon_{0}}\left(x_{c r}\right)$.
Lemma 8. Suppose that a circle homeomorphism $f$ satisfies the conditions ( $a)-(c)$. Let $a, b, c, d \in S^{1}$ and $a \prec b \prec c \prec d \prec$ $a$. Then, there is a constant $Q_{5}>1$ such that

$$
\operatorname{Dcr}(a, b, c, d ; f) \leq Q_{5} .
$$

Proof. By the property of the cross-ratio distortion, we know that $\operatorname{Dcr}(a, b, c, d ; f)=\operatorname{Dr}(a, b, c ; f) \cdot \operatorname{Dr}(d, c, b ; f)$. Now, to prove this lemma, it is enough to show that $\operatorname{Dr}(a, b, c ; f)$ is bounded above. The second ratio distortion $\operatorname{Dr}(d, c, b ; f)$ is analogously estimated. Denote $\mathscr{H}=S^{1} \backslash U_{\epsilon_{0}}\left(x_{c r}\right)$. Consider the following three cases:

(1) $(a, c) \subset \mathscr{H},(2)(a, c) \subset U_{2 \epsilon_{0}}\left(x_{c r}\right)$, and (3) neither (1) nor (2).

If $(a, c) \subset \mathscr{H}$, then

$$
\operatorname{Dr}(a, b, c ; f)=\frac{c-a}{b-a} \cdot \frac{f(b)-f(a)}{f(c)-f(a)} \leq \frac{L}{\ell},
$$

where $L=\sup _{x \in \mathscr{H}} D f(x)$ and $\ell=\inf _{x \in \mathscr{H}} D f(x)$. It is clear that $\ell>0$. Let $(a, c) \subset U_{2 \epsilon_{0}}\left(x_{c r}\right)$. Using Lemma 7, we get

$$
\begin{aligned}
\operatorname{Dr}(a, b, c ; f)= & \frac{c-a}{b-a} \cdot \frac{f(b)-f(a)}{f(c)-f(a)} \\
\leq & \frac{Q_{4}}{Q_{3}} \cdot \frac{\left(c-x_{c r}\right)-\left(a-x_{c r}\right)}{\left(b-x_{c r}\right)-\left(a-x_{c r}\right)} \\
& \times \frac{\left(b-x_{c r}\right)^{3}-\left(a-x_{c r}\right)^{3}}{\left(c-x_{c r}\right)^{3}-\left(a-x_{c r}\right)^{3}} \\
= & \frac{Q_{4}}{Q_{3}} \cdot \operatorname{Dr}(A, B, C ; p),
\end{aligned}
$$

where $A=a-x_{c r}, B=b-x_{c r}, C=c-x_{c r}$, and $p(x)=x^{3}$. It is easy to check that $\operatorname{Dr}(r A, r B, r C ; p)=\operatorname{Dr}(A, B, C ; p)$ for any $r \in R^{1} \backslash\{0\}$. Without any loss of generality, one can assume that $A=1$. Then,

$$
\operatorname{Dr}(1, B, C ; p)=\frac{B^{2}+B+1}{C^{2}+C+1}
$$

Here, there can be four cases: $\left(1^{\prime}\right) A=1<B<C$, $\left(2^{\prime}\right) A=1>0>B>C,\left(3^{\prime}\right) A=1>B>0>C$, and ( $\left.4^{\prime}\right) A=1>B>C>0$. It is easy to check that for all cases $\left(1^{\prime}\right)-\left(4^{\prime}\right)$ the ratio distortion $\operatorname{Dr}(1, B, C ; p)$ is bounded above. Now, consider case (3). It is clear that $c-a>\epsilon_{0}$. From this it is implied that $(f(c)-f(a)) /(c-a) \geq$ const $>0$. Hence, from $(f(b)-f(a)) /(b-a) \leq$ const, (23), and (24), we get the proof of Lemma 8.

Proof of Theorem 4. Let $\mathcal{N}_{m}=\{1,2,3, \ldots, m\}$. We divide the set $\mathcal{N}_{m}$ into three disjoint subsets $\mathscr{D}_{1}, \mathscr{D}_{2}$, and $\mathscr{D}_{3}$ in the following way:

(i) $\mathscr{D}_{1}=\left\{i: i \in \mathcal{N}_{m}\right.$ and $\left.\left(a_{i}, d_{i}\right) \subset U_{2 \epsilon_{0}}\left(x_{c r}\right)\right\}$;

(ii) $\mathscr{D}_{2}=\left\{i: i \in \mathcal{N}_{m}\right.$ and $\left.\left(a_{i}, d_{i}\right) \subset \mathscr{H}\right\}$;

(iii) $\mathscr{D}_{3}=\mathcal{N}_{m} \backslash\left(\mathscr{D}_{1} \cup \mathscr{D}_{2}\right)$. 
We estimate each product $\prod_{i \in \mathscr{D}_{s}} \operatorname{Dcr}\left(a_{i}, b_{i}, c_{i}, d_{i} ; f\right), s=$ 1,2,3 separately. Let $\mathscr{D}_{1}^{1}=\left\{i: i \in \mathcal{N}_{m}\right.$ and $\left.x_{c r} \in\left(a_{i}, d_{i}\right)\right\}$. Then, by Lemma 8, we have

$$
\prod_{i \in \mathscr{D}_{1}^{1}} \operatorname{Dcr}\left(a_{i}, b_{i}, c_{i}, d_{i} ; f\right) \leq Q_{5}^{p} .
$$

Denote $\mathscr{D}_{1}^{2}=\mathscr{D}_{1} \backslash \mathscr{D}_{1}^{1}$; that is, $\mathscr{D}_{1}^{2}=\left\{i: i \in \mathscr{D}_{1}\right.$ and $x_{c r} \notin$ $\left.\left(a_{i}, d_{i}\right)\right\}$. By Lemma 7 , we have

$$
\prod_{i \in \mathscr{D}_{1}^{2}} \operatorname{Dcr}\left(a_{i}, b_{i}, c_{i}, d_{i} ; f\right)<1 .
$$

Let $i \in \mathscr{D}_{2}$. Then, using Lemma 5 , we get

$$
\prod_{i \in \mathscr{D}_{2}} \operatorname{Dcr}\left(a_{i}, b_{i}, c_{i}, d_{i} ; f\right) \leq Q_{2} .
$$

Let $i \in \mathscr{D}_{3}$. Note that the number of intervals does not exceed $2 p$. This and Lemma 8 imply that

$$
\prod_{i \in \mathscr{D}_{3}} \operatorname{Dcr}\left(a_{i}, b_{i}, c_{i}, d_{i} ; f\right) \leq Q_{5}^{2 p} .
$$

Hence, from (26) to (29), the assertion of Theorem 4 follows.

\section{Proof of Main Theorems}

Proof of Theorem 2. The proof of Theorem 2 follows from assertion of Theorem 4 together with the following proposition which was proved by Świątek in [14].

Proposition 9. Let $f$ be a circle homeomorphism with irrational rotation number $\rho$. Assume that $f$ satisfies the cross-ratio inequality with bound $Q_{1}$. Then, there is a circle homeomorphism $H: S^{1} \rightarrow S^{1}$, which conjugates $f$ to the linear rotation $f_{\rho}$. Furthermore, $H$ is quasisymmetric if $\rho$ is of bounded type. If $f$ has at least one critical point of polynomial type, then $H$ is quasisymmetric if and only if $\rho$ is of bounded type.

Before we prove Theorem 3, we formulate two lemmas and use them to prove this theorem. Note that these lemmas also were obtained by Świątek [14].

Lemma 10. Let $f$ be a circle homeomorphism with irrational rotation number. Assume that $f$ satisfies the cross-ratio inequality with bound $Q_{1}$. Then, there is a constant $Q_{6}=$ $Q_{6}\left(Q_{1}, f\right) \geq 1$ such that for every $x \in S^{1}$

$$
Q_{6}^{-1}\left|I_{0}^{n}(x)\right| \leq\left|I_{0}^{n}\left(f^{-q_{n}}(x)\right)\right| \leq Q_{6}\left|I_{0}^{n}(x)\right| .
$$

Lemma 11. Let $f$ be a circle homeomorphism with irrational rotation number. Assume that $f$ satisfies the cross-ratio inequality with bound $Q_{1}$. Then, there is a constant $Q_{7}=$ $Q_{7}\left(Q_{1}, f\right)>0$ such that

$$
\left|I_{0}^{n}\left(x_{c r}\right)\right| \geq Q_{7}\left|I_{0}^{n-1}\left(x_{c r}\right)\right| .
$$

Proof of Theorem 3. It is clear that any two adjacent atoms of $\mathbf{P}_{n}=\mathbf{P}_{n}\left(x_{c r}, f\right)$ can be of the following three types:

$$
\begin{aligned}
& \text { (I) } I_{i}^{n-1}, I_{i+q_{n-1}}^{n-1}, \\
& \text { (II) } I_{i}^{n}, I_{i}^{n-1}, \\
& \text { (III) } I_{i}^{n}, I_{i+q_{n}-q_{n-1}}^{n-1} .
\end{aligned}
$$

In (I), the adjacent atoms are $I$ and $f^{q_{n-1}}(I)$, and in this case by Lemma 10 these intervals are $Q_{6}$-comparable. Consider case (II). Using the property of dynamical partition, it is easy to see that (it suffices to prove it for the linear rotation $f_{\rho}$, which follows from arithmetical properties of $\rho$ ).

$$
I_{0}^{n-1}(x) \supset \bigcup_{s=1}^{k_{n+1}-1} I_{0}^{n}\left(f^{-s q_{n}}(x)\right)
$$

for any $x \in S^{1}$. This together with Lemma 10 implies that

$$
\left|I_{0}^{n-1}(x)\right| \geq\left|I_{0}^{n}\left(f^{-q_{n}}(x)\right)\right| \geq Q_{6}^{-1}\left|I_{0}^{n}(x)\right|,
$$

for any $x \in S^{1}$. If we take $f^{i}\left(x_{c r}\right)$ instead of $x$, we get

$$
\left|I_{i}^{n-1}\right| \geq Q_{6}^{-1}\left|I_{i}^{n}\right| .
$$

Now, we show an opposite inequality to (35). Let us take $I_{i}^{n}, I_{i}^{n-1}$, and $f^{q_{n-1}}\left(I_{i}^{n-1}\right)$. Applying $f^{q_{n}-i}$ to these intervals, we have $f^{q_{n}}\left(I_{0}^{n}\right), f^{q_{n}}\left(I_{0}^{n-1}\right)$, and $f^{q_{n}+q_{n-1}}\left(I_{0}^{n-1}\right)$, respectively. Due to Lemma 10 , the intervals $f^{q_{n}}\left(I_{0}^{n}\right)$ and $I_{0}^{n}$ are $Q_{6}$-comparable. Besides that, by Lemma 11, we have

$$
\left|I_{0}^{n}\right| \geq Q_{7}\left|I_{0}^{n-1}\right| \text {. }
$$

From (34) and (36), it follows that the intervals $I_{0}^{n}$ and $I_{0}^{n-1}$ are $Q_{8}=\max \left\{Q_{6}, Q_{7}^{-1}\right\}$ comparable. Since the intervals $I_{0}^{n}$ and $I_{0}^{n-1}$ are $Q_{8}$-comparable and $I_{0}^{n} \subset f^{q_{n}}\left(I_{0}^{n-1}\right) \subset I_{0}^{n} \cup I_{0}^{n-1}$, the intervals $I_{0}^{n}$ and $f^{q_{n}}\left(I_{0}^{n-1}\right)$ are $1+Q_{8}$-comparable. From this it is implied that the intervals $f^{q_{n}}\left(I_{0}^{n}\right)$ and $f^{q_{n}}\left(I_{0}^{n-1}\right)$ are $Q_{6}(1+$ $\left.Q_{8}\right)$ comparable. Hence, $f^{q_{n}}\left(I_{0}^{n}\right), f^{q_{n}}\left(I_{0}^{n-1}\right)$, and $f^{q_{n}+q_{n-1}}\left(I_{0}^{n-1}\right)$ are $Q_{6}^{2}\left(1+Q_{8}\right)$ comparable.

Since the intervals $I_{i+q_{n-1}}^{n-1}, I_{i}^{n-1}$, and $I_{i}^{n}$ are adjacent on $S^{1}$ and the system of intervals $\left\{f^{s}\left(\left[I_{i+q_{n-1}}^{n-1} \cup I_{i}^{n-1} \cup I_{i}^{n}\right]\right)\right\}, 0 \leq s \leq$ $q_{n}-i$, covers each point of the circle at most three times, by the cross-ratio inequality (12), we have

$$
\begin{gathered}
\frac{\left|f^{q_{n}-i}\left(I_{i}^{n}\right)\right|}{\left|I_{i}^{n}\right|} \cdot \frac{\left|I_{i}^{n}\right|+\left|I_{i}^{n-1}\right|}{\left|f^{q_{n}-i}\left(I_{i}^{n}\right)\right|+\left|f^{q_{n}-i}\left(I_{i}^{n-1}\right)\right|} \\
\cdot \frac{\left|f^{q_{n}-i}\left(I_{i+q_{n-1}}^{n-1}\right)\right|}{\left|I_{i+q_{n-1}}^{n-1}\right|} \cdot \frac{\left|I_{i+q_{n-1}^{n-1}}\right|+\left|I_{i}^{n-1}\right|}{\left|f^{q_{n}-i}\left(I_{i+q_{n-1}}^{n-1}\right)\right|+\left|f^{q_{n}-i}\left(I_{i}^{n-1}\right)\right|} \\
=\prod_{j=0}^{q_{n}-i-1} \operatorname{Dcr}\left(f^{i+2 q_{n-1}+j}\left(x_{c r}\right), f^{i+q_{n-1}+j}\left(x_{c r}\right),\right. \\
\left.f^{i+j}\left(x_{c r}\right), f^{i+q_{n}+j}\left(x_{c r}\right) ; f\right)
\end{gathered}
$$

$\leq Q_{1}(3, f)$. 
Since the intervals $f^{q_{n}}\left(I_{0}^{n}\right), f^{q_{n}}\left(I_{0}^{n-1}\right), f^{q_{n}+q_{n-1}}\left(I_{0}^{n-1}\right)$ are $Q_{6}^{2}\left(1+Q_{8}\right)$-comparable and the intervals $f^{i+q_{n-1}}\left(I_{0}^{n-1}\right)$, $f^{i}\left(I_{0}^{n-1}\right)$ are $Q_{6}$-comparable, and by inequality (37) for constant $Q_{9}=Q_{9}\left(Q_{1}, Q_{6}, Q_{8}\right)>1$, we have

$$
\frac{\left|f^{i}\left(I_{0}^{n}\right)\right|}{\left|f^{i}\left(I_{0}^{n-1}\right)\right|} \geq \frac{\left|f^{i}\left(I_{0}^{n}\right)\right|}{\left|f^{i}\left(I_{0}^{n}\right)\right|+\left|f^{i}\left(I_{0}^{n-1}\right)\right|} \geq Q_{9}^{-1} .
$$

Hence, case (II) follows from (35) and (38). Case (III). By using Lemma 10 twice, we get the comparability of the following intervals: $I_{i+q_{n}}^{n-1}-q_{n-1}, I_{i+q_{n}}^{n-1}$ and $I_{i+q_{n}}^{n-1}, I_{i}^{n-1}$. By case (II), the intervals $I_{i}^{n-1}$ and $I_{i}^{n}$ are comparable and it gives the proof of case (III). Theorem 3 is proved.

\section{Conflict of Interests}

The authors declare that there is no conflict of interests regarding the publication of this paper.

\section{Acknowledgment}

The authors would like to acknowledge the financial support received from the Government of Malaysia under the Research Grant FRGS/2/2013/ST06/UKM/02/2.

\section{References}

[1] I. P. Cornfeld, S. V. Fomin, and Ya. G. Sinaŭ, Ergodic Theory, vol. 245, Springer, Berlin, Germany, 1982.

[2] M. Herman, "Sur la conjugaison différentiable des difféomorphismes du cercle à des rotations," Institut des Hautes Études Scientifiques, vol. 49, pp. 5-234, 1979.

[3] Y. Katznelson and D. Ornstein, "The differentiability of the conjugation of certain diffeomorphisms of the circle," Ergodic Theory and Dynamical Systems, vol. 9, no. 4, pp. 643-680, 1989.

[4] Y. Katznelson and D. Ornstein, "The absolute continuity of the conjugation of certain diffeomorphisms of the circle," Ergodic Theory and Dynamical Systems, vol. 9, no. 4, pp. 681-690, 1989.

[5] K. M. Khanin and Ya. G. Sinaŭ, "Smoothness of conjugacies of diffeomorphisms of the circle with rotations," Russian Mathematical Surveys, vol. 44, no. 1, pp. 69-99, 1989, translation of Uspekhi Matematicheskikh Nauk, vol. 44, pp. 57-82, 1989.

[6] W. de Melo and S. van Strien, One-Dimensional Dynamics, vol. 25, Springer, Berlin, Germany, 1993.

[7] J.-C. Yoccoz, "Il n'y a pas de contre-exemple de Denjoy analytique," Comptes Rendus des Séances de l'Académie des Sciences, vol. 298, no. 7, pp. 141-144, 1984.

[8] J. Graczyk and G. Świątek, "Singular measures in circle dynamics," Communications in Mathematical Physics, vol. 157, no. 2, pp. 213-230, 1993.

[9] Kh. Akhadkulov, "Some circle homeomorphisms with break type singularities," Russian Mathematical Surveys, vol. 61, no. 5, pp. 981-983, 2006.

[10] A. A. Dzhalilov and I. Liousse, "Circle homeomorphisms with two break points," Nonlinearity, vol. 19, no. 8, pp. 1951-1968, 2006.

[11] A. A. Dzhalilov, I. Liousse, and D. Mayer, "Singular measures of piecewise smooth circle homeomorphisms with two break points," Discrete and Continuous Dynamical Systems, vol. 24, no. 2, pp. 381-403, 2009.
[12] A. A. Dzhalilov, D. Maǐer, and U. A. Safarov, "Piecewise-smooth circle homeomorphisms with several break points," Izvestiya, vol. 76, no. 1, pp. 94-112, 2012.

[13] A. Adouani and H. Marzougui, "Singular measures for class P-circle homeomorphisms with several break points," Ergodic Theory and Dynamical Systems, pp. 1-34, 2012.

[14] G. Świątek, "On critical circle homeomorphisms," Boletim da Sociedade Brasileira de Matemática, vol. 29, no. 2, pp. 329-351, 1998.

[15] G. Świątek, "Rational rotation numbers for maps of the circle," Communications in Mathematical Physics, vol. 119, no. 1, pp. 109128, 1988. 


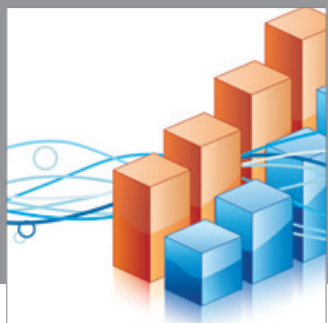

Advances in

Operations Research

mansans

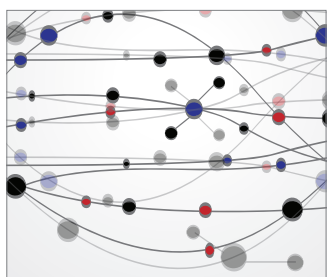

The Scientific World Journal
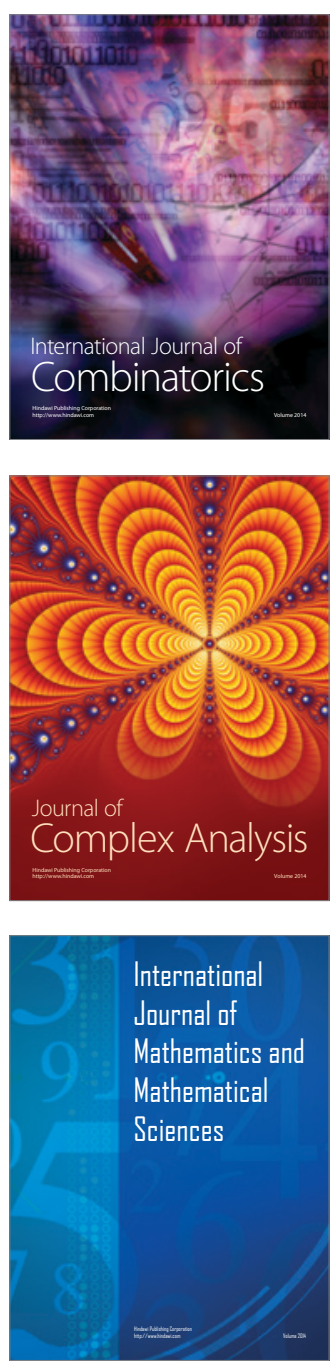
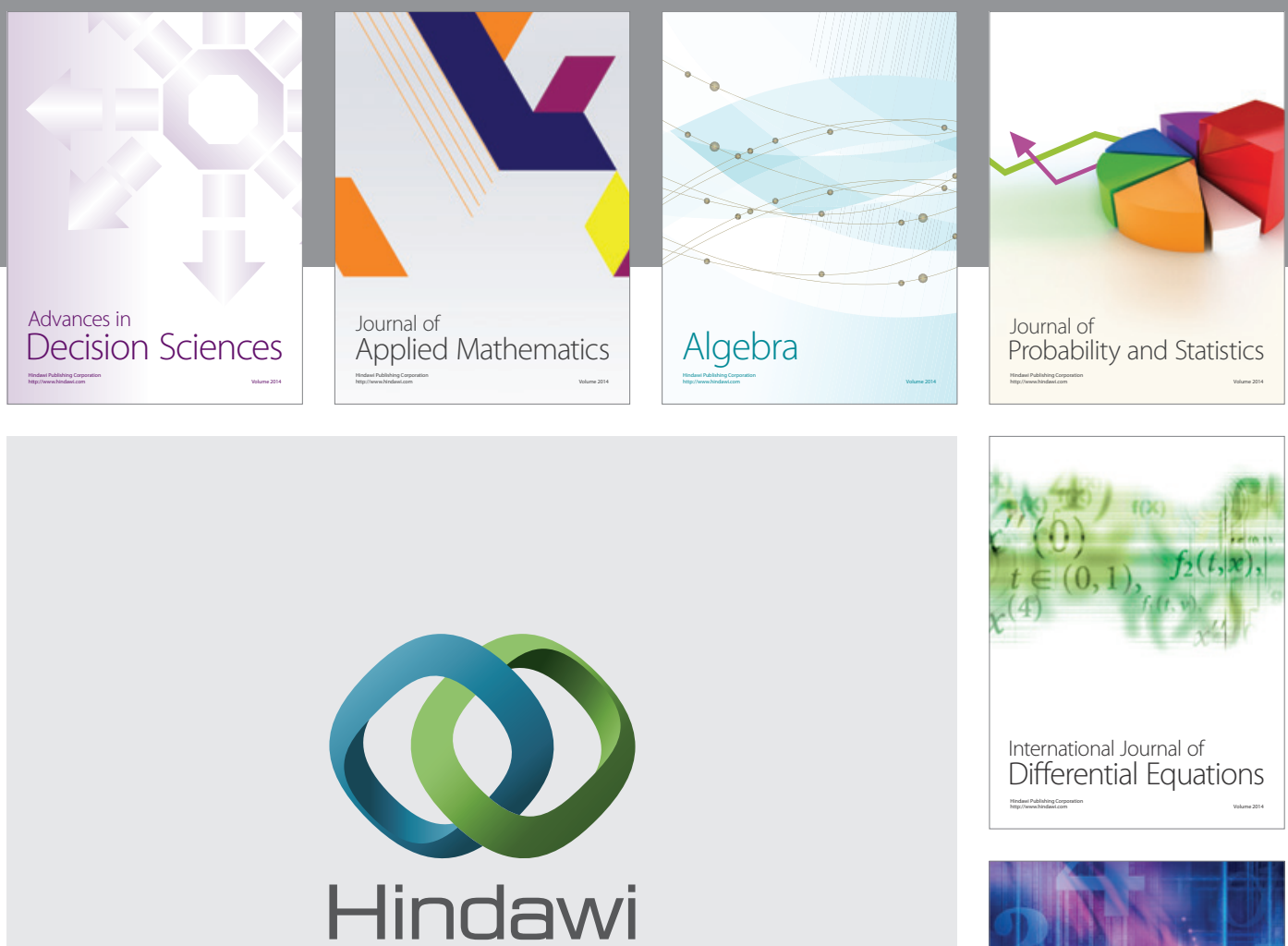

Submit your manuscripts at http://www.hindawi.com
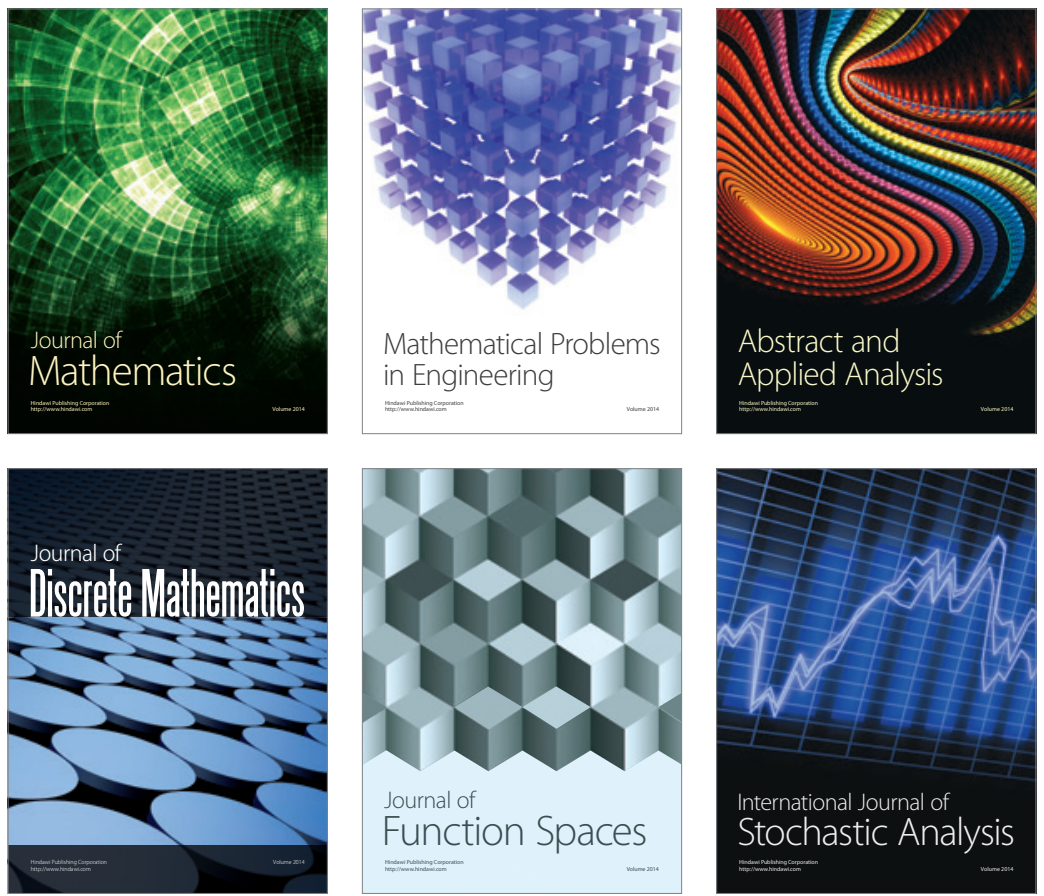

Journal of

Function Spaces

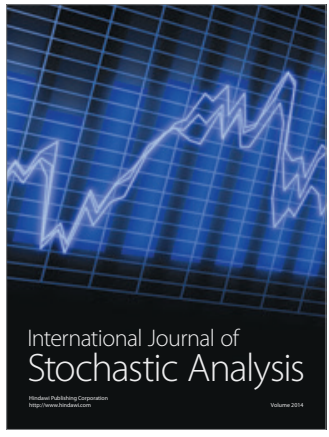

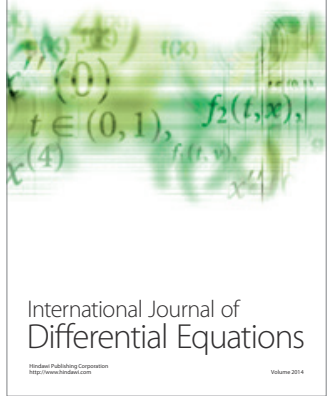
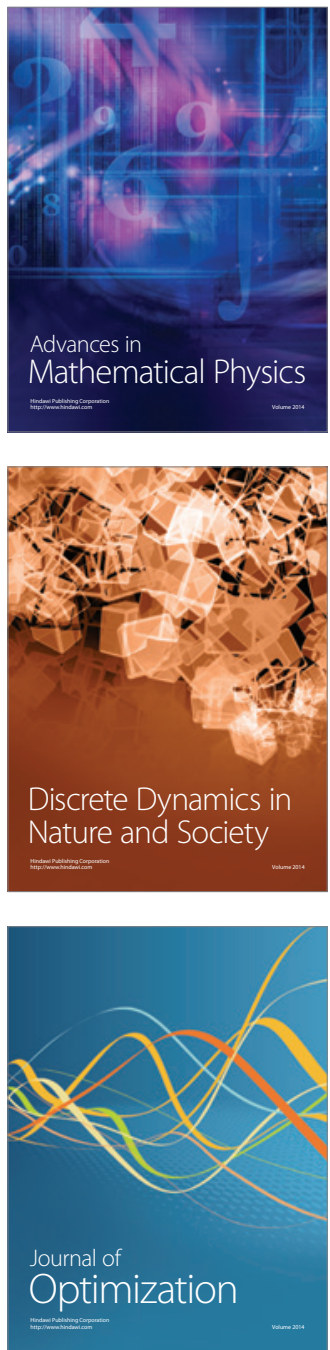